\title{
Accessible Methods Resilience of Power System Electronics to HEMP
}

\author{
Dr. Vladimir Gurevich
}

\begin{abstract}
Protection of modern supersensitive electronics used in power systems (Digital Protective Relays (DPR), control devices and microprocessor-based communication equipment) against High Altitude Electromagnetic Pulse (HEMP) and other Intentional Electromagnetic Interferences (IEMI) is a vital task for today's world [1, 2]. Measures and methods of protection against HEMP and IEMI are well defined and described in technical literature [1, 2]. However, they usually require fundamental change difficult to realize in existing power systems. The article discusses the range of measures and simple technical means designed for existing power systems.
\end{abstract}

\section{INTRODUCTION}

To ensure the reliable full-scale protection against all HEMP components, we need to implement the wide range of technical means and organizational measures, while some of them are difficult to realize in existing power systems. In particular, it is difficult to replace hundreds and thousands of no shielded (or inefficiently shielded) control cables with special-type cables covered with multilayer shields. It is difficult to cut such cables entering control cabinets to interpose special costly filters (especially as they require additional free space in tightly packed cabinet) in series. It is also difficult to replace existing control cabinets containing operating electronics with special designed cabinets and pack them with all internals. As such technical measures certainly ensure highly effective protection against HEMP and are widely used at military control centers, power systems and apparatus; they can also be used in new projects on civil power systems. However, they are too complex and expensive to be used in existing civil power units. Currently, such a high complexity in implementation of protective measures fully prevents power unit staff from realization of any protective measures.

However, can be use less effective but yet much more simple and cheap protection means not capable of providing full protection but ensuring significant attenuation of destructive impacts of HEMP on electronic equipment and increasing its sustainability. Let's discuss such protection methods.

\section{FerRite BEADS}

Ferrite beads are made of two ferrite semi-rings (or semi-cylinders) fixed in two halves of dimidiate plastic body with latch designed to snap both halves on control cable with no need for cable disconnection, see Fig. 1.
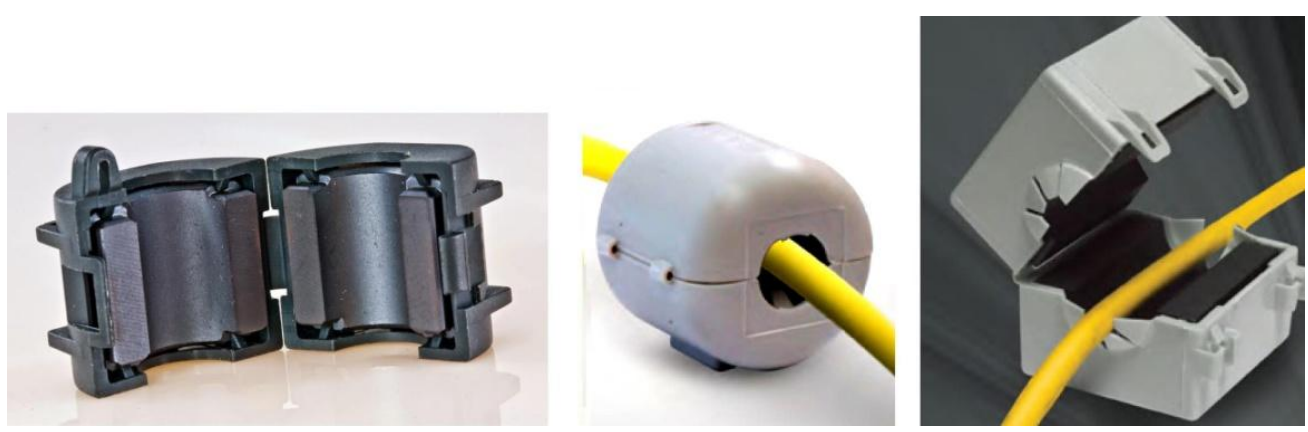

Fig1. Ferrite beads designed for mounting on control cables.

The beads are designed for significant increase in cable inductance against high frequency currents and short pulses. If suitable, such ferrite bead can significantly attenuate current pulse (about $15-20$ $\mathrm{dB}$ ) which is comparable to attenuation effect of reinforced-concrete structure. While this solution seems to be deceptively easy and cheap, such elements are not that simple and require strong 
knowledge of their specifics and parameters [3]. Particularly, [3] demonstrates the rationale for application of three different beads installed in-series on the same cable. However, they are very simple, cheap and available protection devices suitable for every power unit.

\section{Surge Protective Devices}

Since devastating effect of HEMP and other types of IEMI on electronics is caused by generation of high-power high-voltage short pulse penetrating all internal circuits of sensitive electronics, limiting such voltage can be an effective protective measure. Nonlinear zinc-oxide resistors (or metal-oxide varistor - MOV) are the most common elements used for limiting amplitude of surge voltages in AC and DC power circuits, see Fig. 2.
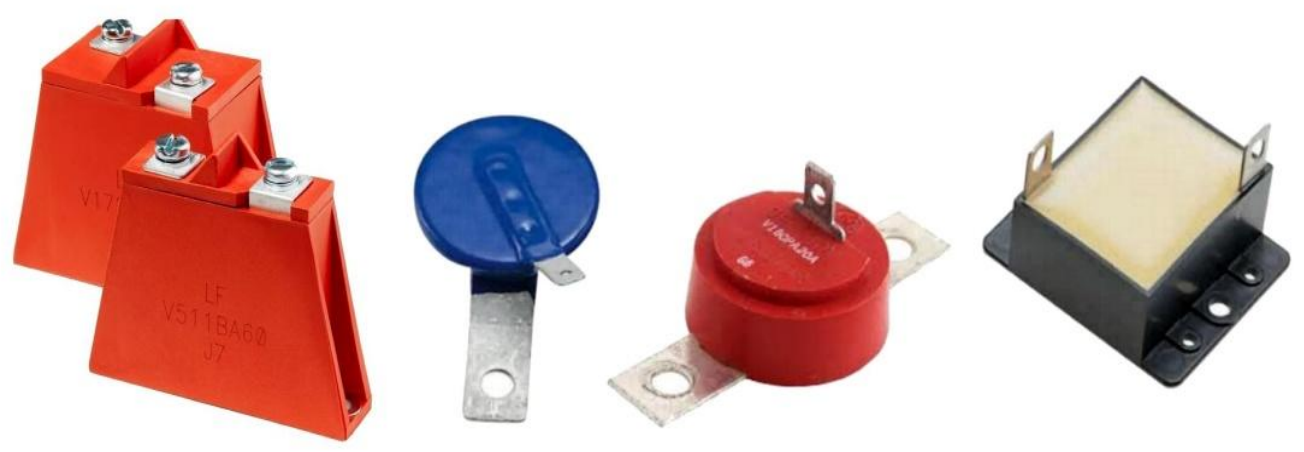

Fig2. High-power zinc-oxide varistors of different types

These well-proven protective elements are suitable for protection of power circuits against switching and lightning surge voltages. Varistors are rated to protect against standard pulse with raising edge of $8 \mu \mathrm{sec}$ and falling edge of $20 \mu \mathrm{sec}$, see Fig. 3 .

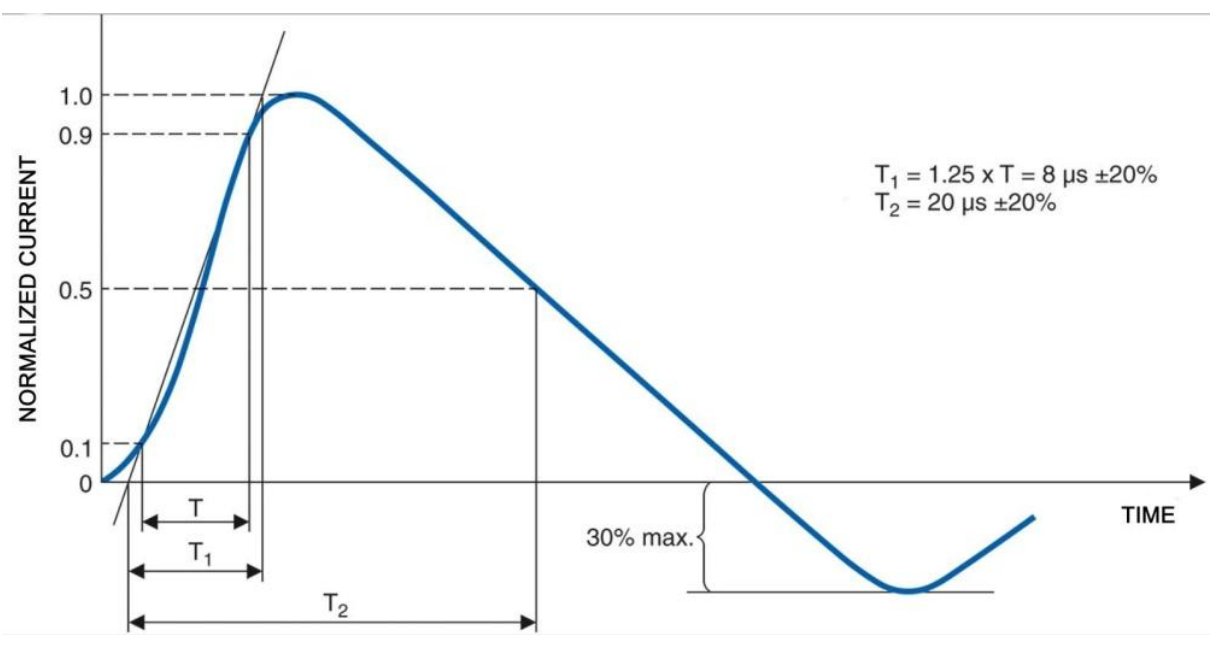

Fig3. Standard lightning pulse of $8 / 20 \mu \mathrm{sec}$.

Available operating voltage (from $250 \mathrm{~V}$ to $500 \mathrm{~V}$ and above) and pulse current (from 10 to $20 \mathrm{kA}$ and above) ranges make varistors perfectly suitable for protection of low voltage AC and DC electric grids and connected equipment against switching and lightning surges. Often enough, varistors are used in special filters designed for protection against HEMP. However, they are not effective against HEMP, see [4 - 5], as HEMP parameters (2/25 ns for voltage and 5/50 ns for current pulse) are significantly different from the parameters of standard lightning surge $(8 / 20 \mu \mathrm{s})$, so the response time of regular varistors is not high enough to limit the very short HEMP pulses.

So called Transient Voltage Suppressor diodes (TVS-diodes) or "suppressors" made based on avalanche diodes act significantly faster. They can effectively protect electronics even against static charges characterized by very short charge pulse currents $(5 / 50 \mathrm{~ns})$ similar to pulses generated during HEMP. Until recently, these elements were used only in low energy circuits due to insufficient power of TVS-diodes. However, over the last years their power has been significantly increased. The manufacturers like Bourns and Littlefuse produce TVS-diodes for operating voltage up to $400 \mathrm{~V}$ and pulse currents up to $10 \mathrm{kA}$, see Fig. 4. 


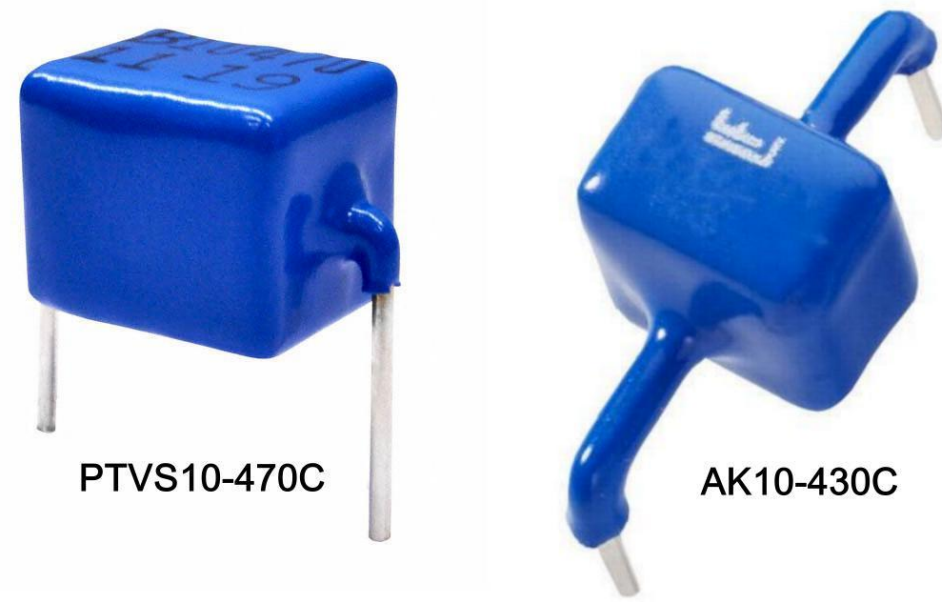

Fig4. The most powerful TVS-diodes (400V, $10 \mathrm{kA})$ manufactured by Bourns (left) and Littlefuse (right) available on the market.

Connected in parallel, several TVS-diodes provide much higher power comparable to the varistor's. In the near future, Littlefuse plans to launch surge arrester model AK20 combined of two structures of TVS-diodes $10 \mathrm{kA}$ each connected in parallel.

For short pulses such as HEMP, the factors negligible upon lightning pulses should be considered. In particular, such negligible characteristics of lightning pulses as the shape and the length of the element terminals conditioning the inductance of the protective element. For 5/50 ns pulse, even the low inductivity of element terminals provides for considerable inductance resulted in its turn in higher residual voltage on protection element upon its operation (so called "clamping voltage"), i.e. in reducing effectiveness of surge protection. Thus, HEMP protection elements should have special terminals. As a rule, element terminals designed for surface mounted devices (SMD) have much lower inductivities than regular wire terminals. This means, that such terminals must be used for TVSdiodes designed for protection against HEMP.

However, there is another technical challenge: TVS-diodes designed for protection of power systems should be installed into the enclosures to be mounted in control and relay cabinets on standard DINrails. Manufacturer of the most powerful TVS-diodes - Littlefuse report that its engineering department is flexible enough to fulfill the requirements of their customers, so probably in the near future they will launch connected in parallel TVS-diodes with SMD terminals installed in the enclosures designed for mounting on DIN-rails.

Power companies must ensure proper installation of such protective elements in cabinets: they should be located as close as possible to protected equipment, the terminals connecting to protective elements should have minimum length and be arranged as short straight-line links of multicore flexible insulated conductor of sufficient section with silver-plated power cores.

\section{Replacement of Permanent Grounding of Electronic Equipment with CONNECTIVE Grounding}

As known, all modern types of DPRs and controllers used in power systems are enclosed in aluminum bodies usually connected to the metal structures of relay cabinets through flexible copper bus see Fig.5.

As a rule, in such devices the electronic circuits internal grounding is connected to the body, i.e. to the external grounding system of the substation (power station). As a HEMP contains both vertical and horizontal components, the external grounding network becomes huge antenna acquiring electromagnetic energy from the wide area and delivering high-voltage pulse directly to the highsensitive electronic components of DPRs and controllers. As [6] shows, proper operation of modern electronic systems, such as DPRs, doesn't depend on grounding availability. It is only required for staff safety as prevents delivery of dangerous potential to the body upon damage of the internal insulation in the electronic equipment. 

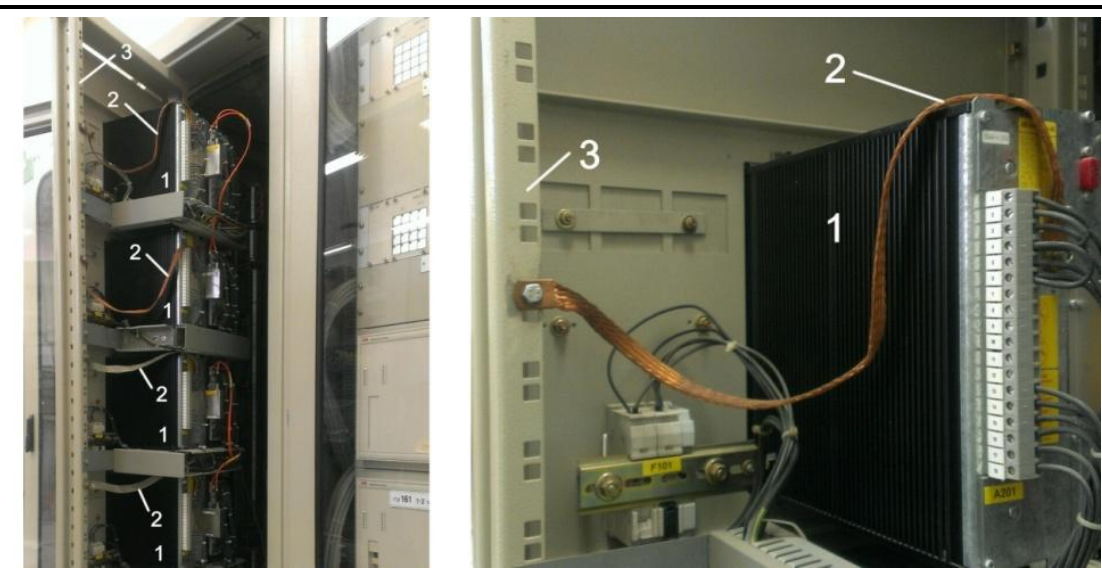

Fig5. DPR grounding in relay cabinets. 1- DPR in metal enclosure, 2 - grounding flexible copper buses, 3 structure element of metal cabinet acting as equipotent surface.

However, the time when the staff is working directly on activated DPR is incommensurably short compared to the total DPR operation time. This results in reasonable idea to provide short-time grounding of DPRs only when the staff is working on them.

From the technical point of view, this idea can be easily realized by so called "position" (or "end" or "limit") switches installed on the relay cabinet door to ground DPR enclosures and controllers when the door opens. Modern position (limit) switches are highly reliable, well-protected against mechanical damage and environmental conditions devices widely available at the market, see Fig. 6 .

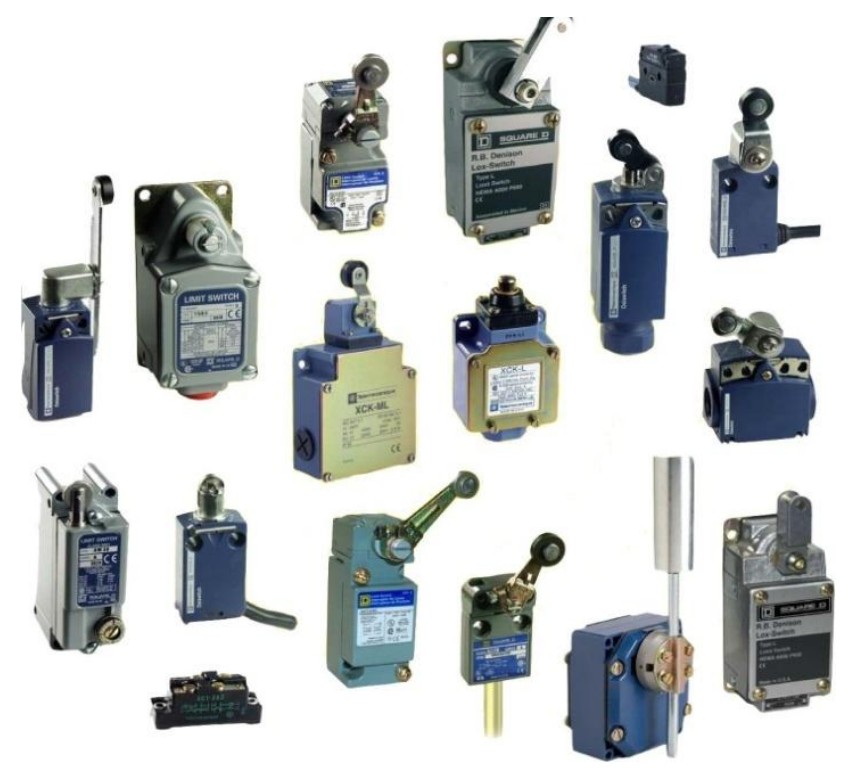

Fig6. Different types of limit switches

They are extensively used in critical industrial systems, road and air transport, military hardware and in staff protection systems. Different types of such switches can changeover currents of $10-16 \mathrm{~A}$, at voltages $400-690 \mathrm{~V}$ and can have different complexity of arrangement: from one NO (NC) contact to several groups of changeover contacts. In order to improve the reliability of DPR enclosure grounding upon opening of relay cabinet door, two limit switches with parallel connected contacts can be installed. To increase the electric strength of contact spacing upon electromagnetic pulse, the switches rated to the maximum operating voltage $(660-690 \mathrm{~V})$ must be used. If there are two similar internal contacts available on the switch, they can be connected in-series.

If permanent grounding is replaced with connective grounding, all metal enclosures of electronics must be connected with flexible copper bus to the common metal bus located in the cabinet and insulated from it with small plastic insulators. Such common bus should be connected to the cabined grounding bar through the limit switch contacts closed upon the door open. In specific cases, if it would seem reasonable, mentioned common metal bus can be wired to the cabinet grounding bus manually during the work in the open cabinet. Such wire must be insulated, permanently connected to the cabinet grounding and be equipped with easily detachable connection to the common metal bus. 


\section{Paradoxical Grounding}

Today all circuits of grounding are designed so that to provide the minimal impedance for short impulse current. For this purpose are created special equipotential surfaces in the control cabinets with the electronic equipment. Connection of the cases of this equipment to equipotential surfaces is carried out by special short, wide and flexible copper buses, fig. 5 . That is take all measures that the high power interferences from grounding system free got into sensitive electronic circuits. However, developing the idea stated above about absence of necessity in grounding of sensitive electronic equipment it is possible to come to a paradoxical conclusion that grounding of the metal cases with electronic equipment inside should be carried out by an opposite paradoxical principle. Such «paradoxical grounding» should provide safety of the personnel at occurrence the dangerous potential of direct or alternative $50 \mathrm{~Hz}$ voltages on the case of the equipment, but should block the power pulse interferences penetration into the equipment from grounding system. The realization of such «paradoxical grounding» is a very simple by increasing an impedance of the conductors connecting the electronic equipment with the bus of grounding for pulse currents. For this purpose the highfrequency choke with high attenuation level for short pulse interference can insert in the connection between case of equipment and bus of grounding. Such choke not has influences on a direct current or an alternatively $50 \mathrm{~Hz}$ current and provide reliable grounding for safety.

\section{IMPROVEMENT OF EleCtromagnetic SHIELDS OF ElECTRONICS CabinetS}

Commonly, modern relay cabinets are equipped with glass doors (see Fig. 7) for better appearance and higher visibility of screens of DPRs and other apparatus installed in the cabinet.

Besides, such cabinets usually don't have floors as dozens of cables are routed into the cabinet through its bottom part. This minimizes shielding properties of such cabinets. However, there are affordable methods to fix it. In the bottom, the free space between the entering cables can be filled with soft wire sponges, several layers of common household metal foil or metalized fabric laid on metal mesh. Such fabric is available from many manufacturers, e.g.: Marktec Inc., Swift Textile Metalizing, Kavon Filter Products and many others.

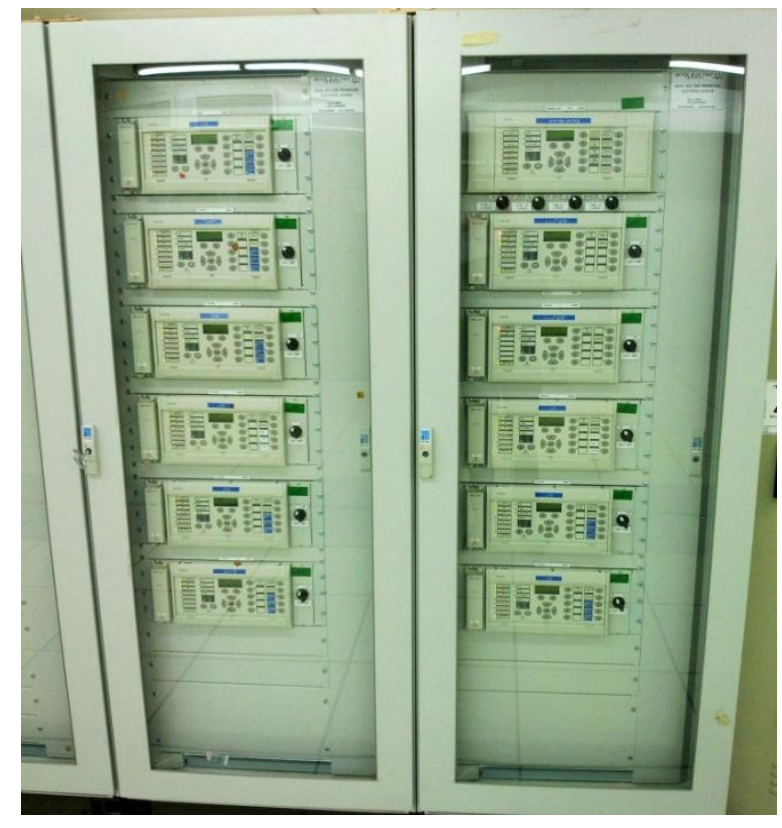

Fig7. Modern relay cabinets with glass doors

Regular glass used in cabinets must be replaced with conductive glass manufactured by Second City Glass, Engineering Glass Products, Swift Glass Company, etc. As an option, the regular glass can be covered with several layers of transparent conductive film manufactured by Acrle Tech., DeWal Industries, Emco Industrial Plastics, etc.

\section{CONCLuSiON}

While the measures described in this article do not provide $100 \%$ protection of power system electronics against HEMP, they can improve its sustainability and immunity at minimum expense and work time expenditures. Also, they do not require significant changes, which is extremely important 
for existing power system equipment. The simplicity and availability of described measures should encourage power system engineering departments to implement the measures for protection of sensitive electronics against HEMP and other IEMI.

In contrast with the new designed high-capable power units, described technical means and organizational arrangements aimed at creation of stock of replacement modules for critical apparatus [7] are actually the only practically available methods to ensure protection against HEMP on existing power systems.

\section{REFERENCES}

[1] Gurevich V. Digital Protective Relays: Problems and Solutions. - Taylor \& Francis Group, Boca Raton, 2011, 404 p.

[2] Gurevich V. Cyber and Electromagnetic Threats in Modern Relay Protection. - Taylor \& Francis Group, Boca Raton, 2015, 205 p.

[3] Gurevich V. The Problem of Correct Choice of Ferrite Beads. - Electrical Engineering \& Electromechanics, 2016, No. 2, pp. $71-73$.

[4] M.-J. Jeng, A. Das, L.-B. Chang, and others, A High Attenuation Electromagnetic Pulse Protector with GDT, MOV and Parallel Coupled BPF on High Thermal Conductivity Substrates. - Progress In Electromagnetics Research Letters, Vol. 33, 73-81, 2012, pp. 73 - 81.

[5] Gurevich V. Lack a Standard for Filters Protecting from Electromagnetic Pulse Makes it Difficult to Develop an Efficient Protection. - Electrical Complexes and Control Systems, 2015, No. 4, pp. $66-70$.

[6] Gurevich V. Functional Grounding of Digital Protective Relays: a Vital Necessity? - Energize, 2015, No. 8, pp. $38-40$.

[7] Gurevich V. Establishment of Inventory of Electronic Equipment Replacement Modules as a Way to Improve the Survivability of the Power System. - International Journal of Electrical and Computer Engineering Systems, 2015, Vol. 6, No. 2, pp. $19-23$.

\section{AUTHOR'S BIOGRAPHY}

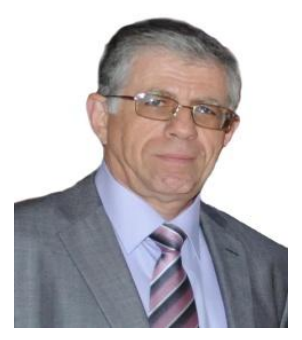

Vladimir I. Gurevich was born in Kharkov, Ukraine, in 1956. He received an M.S.E.E. degree (1978) at the Kharkov Technical University, named after P. Vasilenko, and a Ph.D. degree (1986) at Kharkov National Polytechnic University.

His employment experience includes: teacher, assistant professor and associate professor at Kharkov Technical University, and chief engineer and director of Inventor, Ltd.

In 1994, he arrived in Israel and works today at Israel Electric Corp. as a Senior specialist and Head of section of the Central Electric Laboratory.

$\mathrm{He}$ is the author of more than 200 professional papers and 13 books and holder of nearly 120 patents in the field of electrical engineering and power electronics. In 2006 he was Honorable Professor with the Kharkov Technical University. 